\title{
ARE CARBON CLUSTERS THE CAUSE OF INTERSTELLAR DIFFUSE BANDS?
}

\author{
S P TARAFDAR, K S KRISHNA SWAMY, C BADRINATHAN and \\ D MATHUR \\ Tata Institute of Fundamental Research \\ Homi Bhabha Road \\ Bombay 400 005, INDIA
}

\begin{abstract}
Theoretically determined vertical excitation energies of $C_{5}$ and $C_{7}$ molecules have been shown to agree with the strongest diffuse interstellar band at $4430 \AA$. Several other weak diffuse bands can be identified with vibrational transitions, if $4430 \AA$ band is taken as $0-3$ transition and vibrational constant have a value of $2190 \mathrm{~cm}^{-1}$. The vertical excitation energy of a second electronic transition of $C_{7}$ molecule agrees with diffuse band at $6177 \AA$. This electronic band system may account for diffuse bands at $5778 \AA, 6660 \AA$ and other bands near them.
\end{abstract}

\section{INTRODUCTION}

Since the discovery by Merril (1936), advancement in observations lead to the identification of a large number (about 50) of interstellar diffuse bands, a comprehensive study of which are given by Herbig $(1975,1988)$. The bands, however, still elude identification. Here we compare the wavelengths of theoretically determined vertical transitions of $C_{5}$ and $C_{7}$ molecules with the interstellar diffuse bands.

\section{CALCULATION OF EXCITATION ENERGIES}

The calculation was done in two steps. Firstly, geometrical information regarding equilibrium bond lengths was obtained in all-electron ab initio molecular orbital calculations using unrestricted self-consistent-field Hartree-Fock techniques and optimizing by means of Murtagh-Sargent algorithm (Murtagh and Sargent 1970). The excitation energies and oscillator strengths to low-lying electronic states from ground level were carried out in the second step by means of the semi-empirical HAM/3 (cf. Lindholm and Asbrink 1985) method using the sum of the kinetic energy, electron-electron potential energy, exchange energy and correlation energy as electronic energy. The transition energies and oscillator strengths for $C_{3}, C_{5}$ and $C_{7}$ are given in Table 1. For each transition energies of $C_{5}$ and $C_{7}$ two values 
- one for the determined geometry with different inner and outer bond lengths and the other for the assumed equal bond lengths - are given. The fare agreement between theoretical and experimental $(2.992 \mathrm{ev})$ transition energies for $C_{3}$ suggests that transition energies of $C_{5}$ and $C_{7}$ may be reasonably accurate. However, the same confidence cannot be put on the oscillator strengths which should, therefore, be used for relative comparison.

TABLE 1. Transition Energies and relative oscillator strengths of lowest transitions for $C_{3}, C_{5}$ and $C_{7}$. Two entries for $C_{5}$ and $C_{7}$ are for two different geometrics.

\begin{tabular}{cc|cc|cc}
\hline \multicolumn{2}{c|}{$C_{3}$} & \multicolumn{2}{c|}{$C_{5}$} & \multicolumn{2}{c}{$C_{7}$} \\
\hline \multicolumn{2}{c|}{ Trans En(ev) Rel Osc St } & Trans En(ev) & Rel Osc St & Trans En(ev) & Rel Osc St \\
\hline 2.992 & 0.026 & $2.793-2.764$ & $0.032-0.029$ & $1.962-1.994$ & $0.010-0.008$ \\
- & - & - & - & $2.799-2.816$ & $0.002-0.013$ \\
7.395 & 2.018 & $7.008-6.981$ & $5.99-6.09$ & $6.386-6.357$ & $8.539-9.151$ \\
9.836 & 0.054 & $7.677-7563$ & $0.051-0.003$ & $6.720-6.678$ & $2.231-1.001$ \\
\hline
\end{tabular}

\section{COMPARISON OF THEORETICAL WAVELENGTHS WITH INTERSTEL- LAR DIFFUSE BANDS}

Table 1 shows that both $C_{5}$ and $C_{7}$ have electronic transition near 2.8ev. The wavelengths corresponding to these transitions match well with strongest interstellar diffuse bands at $4428 \AA$. Our calculation does now allow the determination of upper $\left(v^{\prime}\right)$ and lower $\left(v^{\prime \prime}\right)$ vibrational levels of transition nor the vibrational constants $w_{e}^{\prime}$ and $w_{e}^{\prime} x_{e}^{\prime}$. However, if we assume $v^{\prime \prime}=0, v^{\prime}=3, w_{e}^{\prime}=2190 \mathrm{~cm}^{-1}$ and $w_{e}^{\prime} x_{e}^{\prime}=15 \mathrm{~cm}^{-1}$, we can determine the wavelengths for different vibrational transitions and compare them with the observed diffuse bands (Table 2). The observed band wavelengths are put in two columns - one column giving diffuse band wavelengths falling within the theoretical range defined by the geometry and the other outside the theore tical range. Table 2 shows that a large number of diffuse bands fall in the theoretical wavelength range for $C_{5}$ and $C_{7}$. The diffuse bands falling outside but near the theoretical range could arise from other vibrational mode of transition together with the vibrational modes given in the table and if the vibrational constants of the new mode are in the range of $300-500 \mathrm{~cm}^{-1}$ Such values of $w_{e}^{\prime}$ are plausible (cf. Herzberg 1966). Besides the transition near 2.8ev, $C_{7}$ has a strong transition near $2.0 \mathrm{ev}$. The corresponding wavelength range overlaps a number of diffuse bands (Table 2). The wavelengths of theoretical vibrational transitions are determined assuming 2.0 ev transition to be $0-1$ transition, $w_{e}^{\prime}=1170 \mathrm{~cm}^{-1}$ and $w_{\epsilon}^{\prime} x_{\epsilon}^{\prime}=25 \mathrm{~cm}^{-1}$. Table 2 shows that almost all observed interstellar diffuse bands can arise from $C_{7}$ molecule. The observed strengths of $6177 \dot{A}$ and $4428 \dot{A}$ bands are also consistent with their relative theoretical oscillator strengths. Table 1 shows that $C_{5}$ has transitions in the range $1615-1639 \AA$ and $1769-1776 \AA$ and $C_{7}$ in the ranges $1941-1946 \AA$ and $1847-1851 \AA$ besides those discussed above. The $\mathrm{IUE}$ 
TABLE 2. Comparison of diffuse bands with transition wavelengths of $C_{5}$ and $C_{7}$ arising from transition near $2.8 \mathrm{ev}$ and of $C_{7}$ from that near $2 \mathrm{ev}$ (2nd rows).

\begin{tabular}{c|c|c|c|c}
\hline $\begin{array}{c}\text { Vib trans } \\
v^{\prime \prime}-v^{\prime}\end{array}$ & $C_{5}$-trans & $C_{7}$-trans & \multicolumn{2}{|c}{ Observed diffuse bands } \\
\hline $0-3$ & $4439-4485$ & $4403-4429$ & Within $^{*}$ & Outside $^{*}$ \\
$0-2$ & $4895-4952$ & $4851-4883$ & 4882 & 4501 \\
$0-2 \dagger$ & & $5789-5829$ & $5778,5780,5797$ & $4726,4754,4763$ \\
$0-1$ & $5465-5536$ & $5410-5450$ & $5420,5449,5487$ & $5361,58404,5849$ \\
$0-1^{\dagger}$ & & $6171-6217$ & $6177,6195,6203$ & $6314,6353,6362$ \\
$0-0$ & $6196-6287$ & $6126-6178$ & $6234,6264,6283$ & $6010,6042,6113$ \\
$0-0^{\dagger}$ & & $6621-6682$ & $6613,6660,6666$ & $6507,6742,6770$ \\
\hline
\end{tabular}

*Within and outside the theoretical range of wavelengths.

† From $C_{7}$ transition near 2.0ev.

spectra shows that there are no diffuse bands in above wavelength ranges. It is, however, possible, that these bands in UV have disappeared as a result of merger of upper electronic levels of these bands with the continuum in the event of $C_{5}$ and $C_{7}$ residing on a grain surface or in the form small solids. Note that the $C_{5}$ and $C_{7}$ molecules have to reside on a grain surface or in the small solid form for their survival in interstellar diffuse clouds.

\section{REFERENCES}

Herbig G. H. 1975, Astrophys. J. 196, 129.

Herbig G. H. 1988, Astrophys. J. 331, 999.

Herzberg 1966, Molecular Spectra and Molecular Structure, Vol. 3, Van Nostrand Reinhold, New York.

Lindholm E. and Asbrink, L. 1985, Molecular orbitals and their Energies, studies by the semi-empirical HAM Method, Springer-Verlag, Berlin, p. 107.

Merril P. W. 1936, Astrophys. J. 83, 166.

Murtagh B. A. and Sargent R. W. 1970, Comput J. 13, 185. 